\title{
ON THE STEADY-STATE THERMOELASTIC PROBLEM FOR THE HALF-SPACE AND THE THICK PLATE*
}

\author{
BY \\ I. N. SNEDDON AND F. J. LOCKETT \\ The Department of Mathematics, The University of Glasgow
}

1. Introduction. In a recent paper, Sternberg and McDowell [1] discussed the problem of determining the steady-state thermal stresses and displacements in a semiinfinite elastic medium bounded by a plane. In particular they succeeded in proving that the stress field induced by an arbitrary distribution of surface temperatures is plane and parallel to the boundary. The problem was treated by the method of the Green's function.

This paper deals with the determination of the steady-state thermal stresses in both a semi-infinite elastic medium and a thick elastic plate. As in the analysis of Sternberg and McDowell, the problem is treated as one in the classical theory of elasticity. The method of solution employed is that of double Fourier transforms. A general solution, corresponding to an arbitrary temperature field, is obtained in the form of double integrals and it is confirmed that the stress field is plane and parallel to the boundary of the medium. The particular solution corresponding to axially symmetrical temperature fields is deduced and a solution found of the problem in which the surface temperature is uniform over a circular region of exposure and is zero outside. In this special case an expression is given for the difference, $\left|\sigma_{p}-\sigma_{\theta}\right|$, of the principal stresses in terms of tabulated integrals, and the three-dimensional analogue of the isochromatic lines constructed (see Fig. 2).

The corresponding analysis of the steady-state displacements and stresses produced by arbitrary distributions of temperature on the surfaces of a thick plate is then given. It is shown that Sternberg and McDowell's result (that the stress field due to arbitrary surface temperatures is plane and parallel to the boundary) holds for a thick plate as well as for a semi-infinite solid. This result has been derived by McDowell [2], using a method similar to that of the paper [1], and by Muki [3], using a method combining the theory of Fourier series and that of Hankel transforms of integral order. The method developed here is just as simple as these for deriving the general result, and appears to be much more suitable for the discussion of special problems. To illustrate the use of the method, the isochromatic surfaces within a thick plate are constructed for a special distribution of surface temperatures. (see Fig. 3).

2. The basic thermoelastic equations of equilibrium. We consider an elastic body whose boundaries are parallel to the plane $z=0$, and in which is established a temperature field $T+\theta(x, y, z)$, where $T$ is the temperature of the solid in a state of zero stress and strain. We assume that there are no body forces within the solid and that its surfaces are free from traction. If we take a typical length $l$ as our unit of length, the reference

\footnotetext{
*Received April 27, 1959; revised manuscript received July 7, 1959. The results presented in this paper were obtained in the course of an investigation conducted under Contract No. AF 18(600) 1341 with the Air Force Office of Scientific Research of the Air Research and Development Command while the authors were on leave of absence at Duke University, North Carolina.
} 
temperature $T$ as the unit of temperature, and the rigidity modulus $\mu$ as unit of stress, we find [4] that the equations of thermoelastic equilibrium take the dimensionless forms

$$
\nabla^{2} \mathbf{u}+\left(\beta^{2}-1\right) \operatorname{grad} \Delta=b \operatorname{grad} \theta,
$$

where $\mathfrak{u}$ denotes the displacement vector, $\Delta=\operatorname{div} \mathfrak{u}$ is the dilatation, $\beta^{2}=2(1-\nu) /$ $(1-2 v)$ ( $\nu$ being Poisson's ratio), and, in terms of the coefficient of linear expansion of the solid,

$$
b=2(1+\nu) \alpha T^{\prime} /(1-2 \nu) .
$$

The variation of $\theta$ throughout the solid is determined by Laplace's equation

$$
\nabla^{2} \theta=0,
$$

(in the absence of heat sources), and, in this system of units, the relation between the stress tensor $\tau_{i i}$ and the displacement vector $\mathfrak{u}=\left(u_{1}, u_{2}, u_{3}\right)$ is given by the DuhamelNeumann equation

$$
\tau_{i j}=\left[\left(\beta^{2}-2\right) \Delta-b \theta\right] \delta_{i j}+\left(u_{i, i}+u_{i, i}\right) .
$$

In this last equation $u_{i, j}$ denotes $\partial u_{i} / \partial x_{i}$ with the convention that $\left(x_{1}, x_{2}, x_{3}\right) \equiv$ $(x, y, z)$.

To solve the equations of thermoelasticity in this form we introduce the double Fourier transform

$$
f^{*}(\xi, \eta, z)=\frac{1}{2 \pi} \int_{-\infty}^{\infty} \int_{-\infty}^{\infty} f(x, y, z) \exp [i(\xi x+\eta y)] d x d y,
$$

of each physical quantity $f(x, y, z)$ occurring in the problem. The vector equation (1) is then easily seen to be equivalent to the set of three simultaneous ordinary differential equations

$$
\begin{aligned}
\left(D^{2}-\beta^{2} \xi^{2}-\eta^{2}\right) u^{*}-\left(\beta^{2}-1\right) \xi \eta v^{*}-\left(\beta^{2}-1\right) i \xi D w^{*} & =-i b \xi \theta^{*}, \\
-\left(\beta^{2}-1\right) \xi \eta u^{*}+\left(D^{2}-\xi^{2}-\beta^{2} \eta^{2}\right) v^{*}-\left(\beta^{2}-1\right) i \eta D w^{*} & =-i b \eta \theta^{*}, \\
-\left(\beta^{2}-1\right) i \xi D u^{*}-\left(\beta^{2}-1\right) i \eta D v^{*}+\left(\beta^{2} D^{2}-\xi^{2}-\eta^{2}\right) w^{*} & =b D \theta^{*},
\end{aligned}
$$

and the transform of Eq. (2) is

$$
\left(D^{2}-\zeta^{2}\right) \theta^{*}=0 \text {, }
$$

where we have written $\zeta=\left|\left(\xi^{2}+\eta^{2}\right)^{1 / 2}\right|$ and used $D$ to denote the differential operator $d / d z$. It can be shown that the solution of this set of simultaneous ordinary differential equations may be put in the form

$$
\begin{aligned}
\theta^{*} & =E e^{-\zeta z}+E^{\prime} e^{\zeta z}, \\
u^{*} & =\left(A_{1}+P \xi z\right) e^{-\zeta z}+\left(A_{1}^{\prime}+P^{\prime} \xi z\right) e^{\zeta z}, \\
v^{*} & =\left(A_{2}+P \eta z\right) e^{-\zeta z}+\left(A_{2}^{\prime}+P^{\prime} \eta z\right) e^{\zeta z}, \\
w^{*} & =\left(A_{3}-i P \zeta z\right) e^{-\zeta z}+\left(A_{3}^{\prime}-i P^{\prime} \zeta z\right) e^{\zeta z},
\end{aligned}
$$

where the constants $A_{1}, A_{2}, A_{3}, A_{1}^{\prime}, A_{2}^{\prime}, A_{3}^{\prime}, E, E^{\prime}$ are arbitrary and $P, P^{\prime}$ are given in terms of them by the equations

$$
\begin{aligned}
P & =\left[i b E-\left(\beta^{2}-1\right)\left(\xi A_{1}+\eta A_{2}-i \zeta A_{3}\right)\right] \zeta^{-1}\left(\beta^{2}+1\right)^{-1}, \\
P^{\prime} & =\left[i b E^{\prime}-\left(\beta^{2}-1\right)\left(\xi A_{1}^{\prime}+\eta A_{2}^{\prime}-i \zeta A_{3}^{\prime}\right)\right] \zeta^{-1}\left(\beta^{2}+1\right)^{-1} .
\end{aligned}
$$


The expressions given by Eqs. (4) and (5) satisfy the basic equations of thermoelastic equilibrium in the transformed form. To obtain the solution of the original equations we make use of the Fourier inversion theorem.

$$
f(x, y, z)=\frac{1}{2 \pi} \int_{-\infty}^{\infty} \int_{-\infty}^{\infty} f^{*}(\xi, \eta, z) \exp [-i(\xi x+\eta y)] d \xi d \eta,
$$

to obtain the expression for a physical quantity in terms of its Fourier transform. The solutions (4) and (5) are, of course, suitable only for the discussion of problems in which the solid region under consideration is bounded by planes normal to the $z$-axis.

3. Solution for the half-space. If we assume that the components of the displacement vector, and the temperature each tend to zero as $z \rightarrow \infty$, the solution for the half-space $z \geq 0$ is

$$
u^{*}=\left(A_{1}+\xi P z\right) e^{-\zeta z}, \quad v^{*}=\left(A_{2}+\eta P z\right) e^{-\zeta z}, \quad w^{*}=\left(A_{3}-i \zeta P z\right) e^{-\zeta z},
$$

where $A_{1}, A_{2}, A_{3}$ denote arbitrary constants,

$$
\theta^{*}=E e^{-\zeta z},
$$

and $P$ is given by Eq. (6). The Fourier transforms of the stress components $\tau_{x z}, \tau_{y z}, \sigma_{z}$ are given then by the equations

$$
\begin{aligned}
\tau_{x z}^{*} & =\left(-\zeta A_{1}-\xi \zeta P z+\xi P-i \xi A_{3}-\xi \zeta P z\right) e^{-\zeta z}, \\
\tau_{\nu z}^{*} & =\left(-\zeta A_{2}-\eta \zeta P z+\eta P-i \eta A_{3}-\eta \zeta P z\right) e^{-\zeta z}, \\
\sigma_{z}^{*} & =\left[-i\left(\beta^{2}-2\right)\left(\xi A_{1}+\xi^{2} P z+\eta A_{2}+\eta^{2} P z\right)+\beta^{2}\left(-\zeta A_{3}+i \zeta^{2} P z-i \zeta P\right)-b E\right] e^{-\zeta z} .
\end{aligned}
$$

If, then, we impose the boundary conditions

$$
\theta=\phi(x, y), \quad \tau_{x z}=\tau_{y z}=\sigma_{z}=0,
$$

on the plane $z=0$, we find that

$$
E=\phi^{*}(\xi, \eta),
$$

where $\phi^{*}(\xi, \eta)$ denotes the double Fourier transform of $\phi(x, y)$, and that

$$
\left(A_{1}, A_{2}, A_{3}\right)=i b \phi^{*}\left[2\left(\beta^{2}-1\right) \zeta^{2}\right]^{-1}(\xi, \eta, i \zeta) .
$$

For these values of the arbitrary constants $A_{1}, A_{2}, A_{3}$ it is readily seen, from Eq. (6), that $P=0$ so that the solution (8) can be written in the form

$$
\mathbf{u}^{*}=i b \phi^{*} e^{-\zeta z}\left[2\left(\beta^{2}-1\right) \zeta^{2}\right]^{-1}(\xi, \eta, i \zeta) .
$$

For this solution it is easily shown that

$$
\tau_{x z}^{*}=\tau_{y z}^{*}=\sigma_{z}^{*}=0,
$$

for all positive values of $z$, showing that the stress field is plane and parallel to the boundary in agreement with the result of Sternberg and McDowell.

If we invert Eq. (11) by the Fourier inversion theorem, we find that the displacement vector is given by the equation

$$
\mathbf{u}=\frac{b}{4 \pi\left(\beta^{2}-1\right)} \int_{-\infty}^{\infty} \int_{-\infty}^{\infty}(i \xi, i \eta,-\zeta) \exp [-i(\xi x+\eta y)-\zeta z] \frac{\phi^{*}(\xi, \eta) d \xi d \eta}{\xi^{2}+\eta^{2}}
$$


In the case in which the prescribed surface temperature $\phi(x, y)$ is axially symmetrical, so that $\phi(x, y)=\phi(\rho)$, where $\rho=\left(x^{2}+y^{2}\right)^{1 / 2}$, we find that

$$
\phi^{*}(\xi, \eta)=(2 \pi)^{-1} \int_{0}^{2 \pi} d \vartheta \int_{0}^{\infty} \rho \phi(\rho) \exp [i \zeta \rho \cos (\vartheta-\chi)] d \rho,
$$

where we have written $\xi=\zeta \cos \chi, \eta=\zeta \sin \chi, x=\rho \cos \vartheta, y=\rho \sin \vartheta$. We therefore find that, in this case, $\phi^{*}(\xi, \eta)=\phi^{*}(\zeta)$, where $\phi^{*}(\zeta)$ denotes the zero-order Hankel transform of the function $\phi(\rho)$ defined by the equation

$$
\phi^{*}(\zeta)=\int_{0}^{\infty} \rho J_{0}(\rho \zeta) \phi(\rho) d \rho
$$

Denoting the component of the vector $u$ in the $\rho$-direction by $u_{\rho}$ we find that

$$
\begin{aligned}
& u_{\rho}=u \cos \vartheta+v \sin \vartheta \\
& =b\left[4 \pi\left(\beta^{2}-1\right)\right]^{-1} \int_{0}^{\infty} \phi^{*}(\zeta) e^{-\zeta z} d \zeta \int_{0}^{2 \pi} i \cos (\vartheta-\chi) \exp [-i \zeta \rho \cos (\vartheta-\chi)] d \phi \\
& =\frac{1}{2} b\left(\beta^{2}-1\right)^{-1} \int_{0}^{\infty} \phi^{*}(\zeta) J_{1}(\rho \zeta) e^{-\zeta z} d \zeta .
\end{aligned}
$$

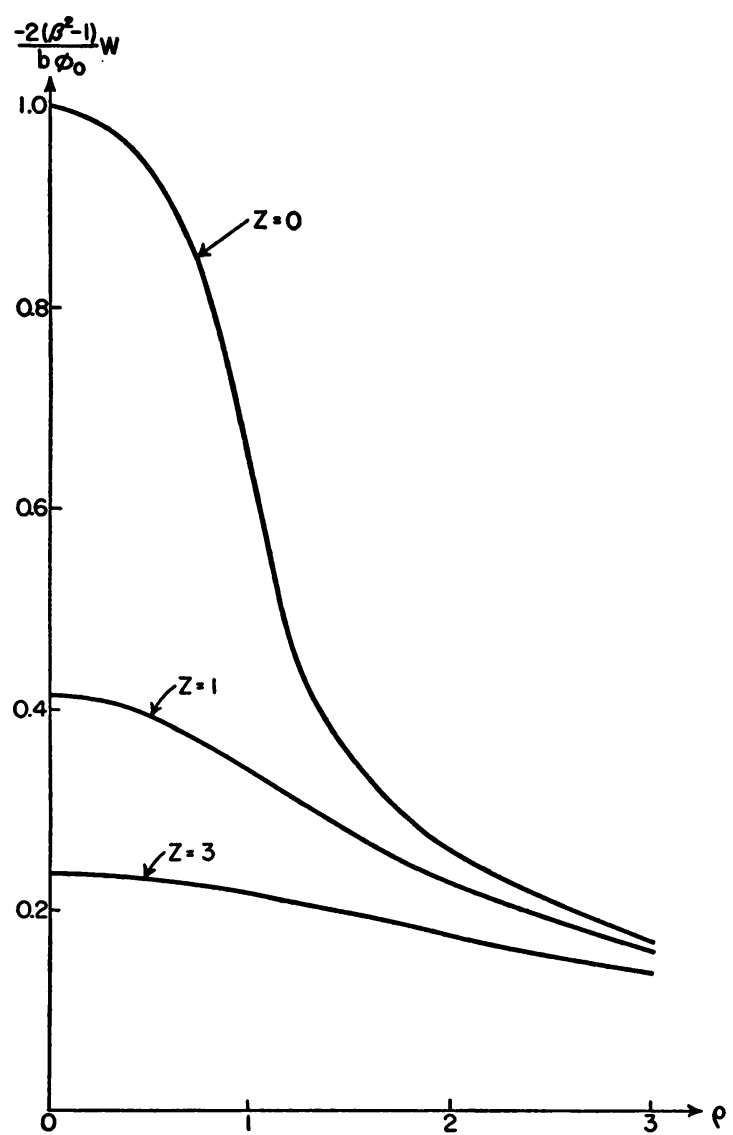


Also the component in the $z$-direction is given by

$$
w=-\frac{1}{2} b\left(\beta^{2}-1\right)^{-1} \int_{0}^{\infty} \phi^{*}(\zeta) J_{0}(\rho \zeta) e^{-\zeta z} d \zeta .
$$

As a special case of the use of these formulae, we consider the problem which is considered in some detail by Sternberg and McDowell, namely that in which

$$
\phi(\rho)=\left\{\begin{array}{rr}
\phi_{0}, & 0<\rho<1 ; \\
0, & \rho>1,
\end{array}\right.
$$

in which case $\phi^{*}(\zeta)=\phi_{0} J_{1}(\zeta) / \zeta$ and, in the notation of [5] we have

$$
u=\frac{1}{2} b \phi_{0}\left(\beta^{2}-1\right)^{-1} J(1,1 ;-1), \quad w=-\frac{1}{2} b \phi_{0}\left(\beta^{2}-1\right)^{-1} J(1,0 ;-1),
$$

where

$$
J(\mu, \nu ; \lambda)=\int_{0}^{\infty} J_{\mu}(\zeta) J_{\nu}(\rho \zeta) e^{-\zeta z} \zeta^{\lambda} d \zeta .
$$

The integrals $J(1,1 ;-1)$ and $J(1,0 ;-1)$ have been tabulated for ranges of values of $\rho$ and $z$ in Tables 9,10 and 11 on pp. 545-546 of [5], so that it is an easy matter to calculate the components of the displacement vector at any point. For example, Fig. 1 shows the variation of the normal component, $w$, of the displacement in planes parallel to the boundary.

Using the formulae developed in [5], it is a simple matter to show that the equations (15) are in agreement with the expressions given by Sternberg and McDowell.

It is of more direct interest to calculate the difference of the principal stresses. In our system of units, we have

$$
\sigma_{\rho}-\sigma_{\vartheta}=2\left(\frac{\partial u_{\rho}}{\partial \rho}-\frac{u_{\rho}}{\rho}\right),
$$

so that, in the general case

$$
\sigma_{\rho}-\sigma_{\vartheta}=b\left(\beta^{2}-1\right)^{-1} \int_{0}^{\infty} \zeta \phi^{*}(\zeta)\left[J_{0}(\rho \zeta)-2 J_{1}(\rho \zeta) /(\rho \zeta)\right] e^{-\zeta z} d \zeta,
$$

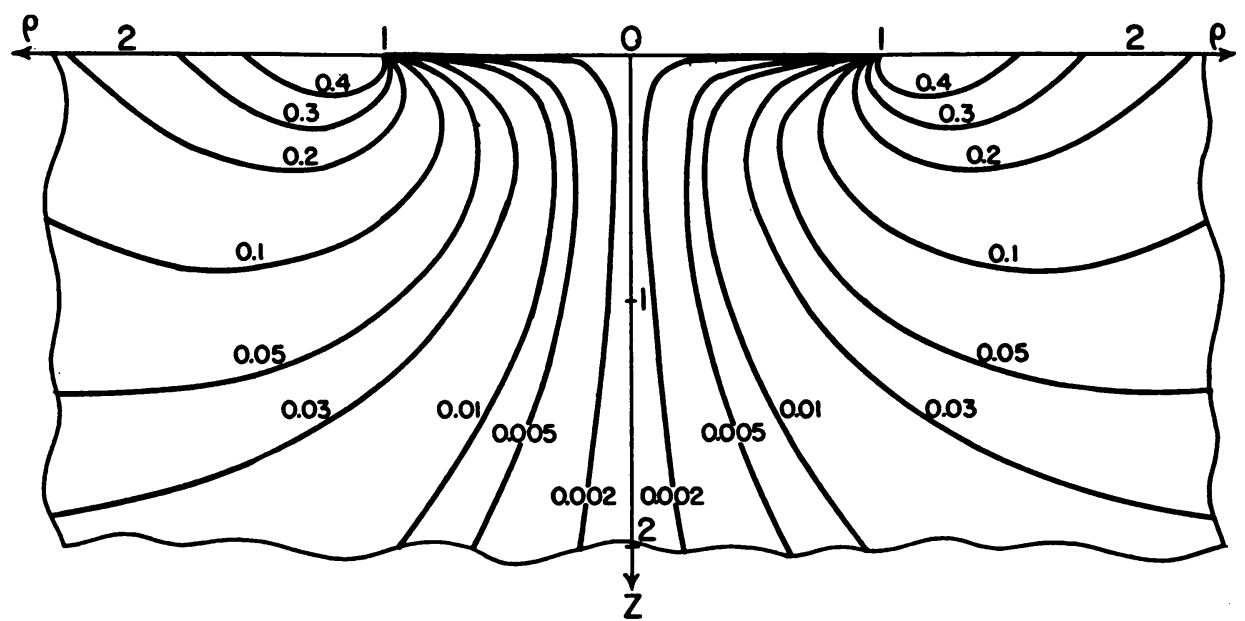

Fra. 2. Section by the plane $\vartheta=$ constant of the surface $\left|\sigma_{\rho}-\sigma_{\vartheta}\right|=$ constant, in a semi-infinite solid. The numbers refer to values of $\left(\beta^{2}-1\right)\left(\sigma_{\vartheta}-\sigma_{\rho}\right) / b \phi_{0}$. 
and, in the case in which $\zeta \phi^{*}(\zeta)=\phi_{0} J_{1}(\zeta)$,

$$
\sigma_{\rho}-\rho_{\vartheta}=b \phi_{0}\left(\beta^{2}-1\right)^{-1}[J(1,0 ; 0)-2 J(1,1 ;-1) / \rho] .
$$

The values of this stress difference at a grid of points in the $z \rho$-plane can be calculated easily from Tables 6 and 11 of [5], and it is then a simple matter to draw out the lines joining points with the same value of this stress difference. The resulting set of curves will be curves obtained by cutting surfaces of equal maximum shearing stress by a plane $\vartheta=$ constant, and will correspond to the isochromatic lines of two-dimensional elasticity. These contours are shown in Fig. 2 for the simple problem we have considered here.

4. Solution for a thick plate. The general solutions (5) can be written in a form which is more suitable to plate problems, in which the elastic solid is bounded by the planes $z= \pm d$. The displacements due to the temperature distribution whose transform is

$$
\theta^{*}=E \cosh (\zeta z)+E^{\prime} \sinh (\zeta z)
$$

are given by the equations

$$
\begin{aligned}
u^{*} & =\left(A_{1}+P^{\prime} \xi z\right) \cosh (\zeta z)+\left(A_{1}^{\prime}+P \xi z\right) \sinh (\zeta z), \\
v^{*} & =\left(A_{2}+P^{\prime} \eta z\right) \cosh (\zeta z)+\left(A_{2}^{\prime}+P \eta z\right) \sinh (\zeta z), \\
w^{*} & =\left(A_{3}+i P^{\prime} \zeta z\right) \sinh (\zeta z)+\left(A_{3}^{\prime}+i P \zeta z\right) \cosh (\zeta z),
\end{aligned}
$$

where

$$
\begin{aligned}
P^{\prime} & =\left(\beta^{2}-1\right)\left(\xi A_{1}^{\prime}+\eta A_{2}^{\prime}+i \zeta A_{3}^{\prime}\right) /\left(\beta^{2}+1\right) \zeta-i b E^{\prime} /\left(\beta^{2}+1\right) \zeta, \\
P & =\left(\beta^{2}-1\right)\left(\xi A_{1}+\eta A_{2}+i \zeta A_{3}\right) /\left(\beta^{2}+1\right) \zeta-i b E /\left(\beta^{2}+1\right) \zeta .
\end{aligned}
$$

The components $\tau_{x z}, \tau_{y z}, \sigma_{z}$ of the stress tensor associated with this displacement field are given by the equations

$$
\begin{aligned}
\tau_{x z}^{*}= & \left(\zeta A_{1}-i \xi A_{3}+P \xi\right) \sinh (\zeta z)+2 P \xi \xi z \cosh (\zeta z)+\left(\zeta A_{1}^{\prime}-i \xi A_{3}^{\prime}+P^{\prime} \xi\right) \cosh (\zeta z) \\
& +2 P^{\prime} \xi \zeta z \sinh (\zeta z), \\
\tau_{\nu z}^{*}= & \left(\zeta A_{2}-i \eta A_{3}+P \eta\right) \sinh (\zeta z)+2 P \eta \zeta z \cosh (\zeta z) \\
& +\left(\zeta A_{2}^{\prime}-i \eta A_{3}^{\prime}+P^{\prime} \eta\right) \cosh (\zeta z)+2 P^{\prime} \eta \zeta z \sinh (\zeta z) . \\
\sigma_{z}^{*}= & -i\left(\beta^{2}-2\right)\left[\left(A_{1} \xi+A_{2} \eta\right) \cosh (\zeta z)+P \zeta^{2} z \sinh (\zeta z)\right]-b E \cosh (\zeta z) \\
& +\beta^{2} A_{3} \zeta \cosh (\zeta z)+\beta^{2} i \zeta P \cosh (\zeta z)+\beta^{2} i \zeta^{2} z P \sinh (\zeta z) \\
& -i\left(\beta^{2}-2\right)\left[\left(A_{1}^{\prime} \xi+A_{2}^{\prime} \eta\right) \sinh (\zeta z)+P^{\prime} \zeta^{2} z \cosh (\zeta z)\right]-b E^{\prime} \sinh (\zeta z) \\
& +\beta^{2} A_{3}^{\prime} \zeta \sinh (\zeta z)+\beta^{2} i \zeta P^{\prime} \sinh (\zeta z)+\beta^{2} i \zeta^{2} z P^{\prime} \cosh (\zeta z) .
\end{aligned}
$$

Since the right-hand sides of these equations consist of the sum of an odd and an even part, the boundary conditions $\sigma_{z}=\tau_{x z}=\tau_{y z}=0$, on $z= \pm d$, lead to three pairs of equations of the type $a+b=0, a-b=0$, which imply that $a=0, b=0$. In this way we get the three equations 


$$
\begin{gathered}
\left(\zeta A_{1}-i \xi A_{3}+P \xi\right) \sinh \zeta d+2 P \xi \zeta d \cosh \zeta d=0, \\
\left(\zeta A_{2}-i \eta A_{3}+P \eta\right) \sinh \zeta d+2 P \eta \zeta d \cosh \zeta d=0,
\end{gathered}
$$

$-i\left(\beta^{2}-2\right)\left[\left(A_{1} \xi+A_{2} \eta\right) \cosh \zeta d+P \zeta^{2} d \sinh \zeta d\right]-b E \cosh \zeta d+\beta^{2} A_{3} \zeta \cosh \zeta d$

$$
+\beta^{2} i \zeta P \cosh \zeta d+\beta^{2} i \zeta^{2} d P \sinh \zeta d=0,
$$

from which, with the help of the second of equations (21), we find that

$$
\left(A_{1}, A_{2}, A_{3}\right)=\frac{1}{2} i b E \zeta^{-2}\left(\beta^{2}-1\right)^{-1}(\xi, \eta,-i \zeta),
$$

and, in turn from these, that $P=0$.

We get a similar set of equations with the $A_{i}$ replaced by $A_{i}^{\prime}$ and $E$ replaced by $E^{\prime}$. In this way we obtain the expressions

$$
\begin{aligned}
u^{*} & =\frac{1}{2} i b \xi \zeta^{-2}\left(\beta^{2}-1\right)^{-1}\left(E \cosh \zeta z+E^{\prime} \sinh \zeta z\right), \\
v^{*} & =\frac{1}{2} i b \eta \zeta^{-2}\left(\beta^{2}-1\right)^{-1}\left(E \cosh \zeta z+E^{\prime} \sinh \zeta z\right), \\
w^{*} & =\frac{1}{2} b \zeta^{-1}\left(\beta^{2}-1\right)^{-1}\left(E \sinh \zeta z+E^{\prime} \cosh \zeta z\right),
\end{aligned}
$$

for the Fourier transforms of the components of the displacement vector. Substituting the values for the constants into the equations (22), we find that $\tau_{x z}^{*}=\tau_{y z}^{*}=\sigma_{z}^{*}=0$, $-d \leq z \leq d$, so that the Sternberg-McDowell result that the stress field induced by an arbitrary distribution of surface temperature is plane and parallel to the boundary holds for a thick plate as well as for a semi-infinite solid.

As in the case of the half-space we can easily derive the solution in the case in which the temperature field is axially symmetrical. To illustrate the procedure, we shall consider the simple situation in which

$$
\theta=\phi(\rho), \quad \text { on } z=+d, \quad \theta=0 \text { on } z=-d .
$$

For this distribution of surface temperature, we find that

$$
\begin{gathered}
\theta=\int_{0}^{\infty} \zeta \phi^{*}(\zeta) \sinh [\zeta(z+d)] \operatorname{cosech}(2 \zeta d) J_{0}(\zeta \rho) d \zeta \\
u_{\rho}=\frac{1}{2} b\left(\beta^{2}-1\right)^{-1} \int_{0}^{\infty} \phi^{*}(\zeta) \sinh [\zeta(z+d)] \operatorname{cosech}(2 \zeta d) J_{1}(\zeta \rho) d \zeta \\
w=\frac{1}{2} b\left(\beta^{2}-1\right)^{-1} \int_{0}^{\infty} \phi^{*}(\zeta) \cosh [\zeta(z+d)] \operatorname{cosech}(2 \zeta d) J_{0}(\zeta \rho) d \zeta .
\end{gathered}
$$

Our unit of length $(l)$ is, as yet, unspecified. If we now take $l=d$, so that all lengths are measured as ratios of half the thickness of the plate, we find that Eqs. (25) to (27) assume the simpler forms

$$
\begin{gathered}
\theta=\int_{0}^{\infty} \zeta \phi^{*}(\zeta) \sinh [\zeta(z+1)] \operatorname{cosech}(2 \zeta) J_{0}(\zeta \rho) d \zeta \\
u_{\rho}=\frac{1}{2} b\left(\beta^{2}-1\right)^{-1} \int_{0}^{\infty} \phi^{*}(\zeta) \sinh [\zeta(z+1)] \operatorname{cosech}(2 \zeta) J_{1}(\zeta \rho) d \zeta, \\
w=\frac{1}{2} b\left(\beta^{2}-1\right)^{-1} \int_{0}^{\infty} \phi^{*}(\zeta) \cosh [\zeta(z+1)] \operatorname{cosech}(2 \zeta) J_{0}(\zeta \rho) d \zeta
\end{gathered}
$$


For any given distribution of temperature on the upper surface of the plate, we can calculate the zero-order Hankel transform $\phi^{*}(\zeta)$ of the temperature $\phi(\rho)$, and, inserting this transform in Eqs. (25a) to (27a), calculate the temperature field and the displacement field within the plate. In the general case, the evaluation of these integrals would be a pretty complicated process, because of the occurrence of the factor cosech $(2 \zeta)$ in the integrand. By suitably choosing the function $\phi(\rho)$ we can, however, obtain integrals which can be easily evaluated, and obtain the solution of a representative problem.

For example, if we take the distribution of temperature on the upper surface of the plate to be given by the function

$$
\phi(\rho)=\left[\left(k^{2}-4\right)^{2} \theta_{0} / 8 k\right]\left\{(k-2)\left[\rho^{2}+(k-2)^{2}\right]^{-3 / 2}-(k+2)\left[\rho^{2}+(k+2)^{2}\right]^{-3 / 2}\right\},
$$

then the temperature at the point $z=d, \rho=0$, or, in our system of units, $z=1, \rho=0$, is $\theta_{0}$, and the zero-order Hankel transform is

$$
\phi^{*}(\rho)=\left[\frac{1}{4}\left(k^{2}-4\right)^{2} \theta_{0} / k\right] e^{-k \zeta} \sinh (2 \zeta), \quad(k>2) .
$$

If, now, we substitute the expression (29) into Eq. (25a) and evaluate the simple integrals so obtained, we find that the temperature field is given by the equation

$$
\begin{aligned}
\theta=\left[\left(k^{2}-4\right)^{2} \theta_{0} / 8 k\right]\left\{( k - 1 - z ) \left[\rho^{2}\right.\right. & \left.+(k-1-z)^{2}\right]^{-3 / 2} \\
& \left.-(k+1+z)\left[\rho^{2}+(k+1+z)^{2}\right]^{-3 / 2}\right\} .
\end{aligned}
$$

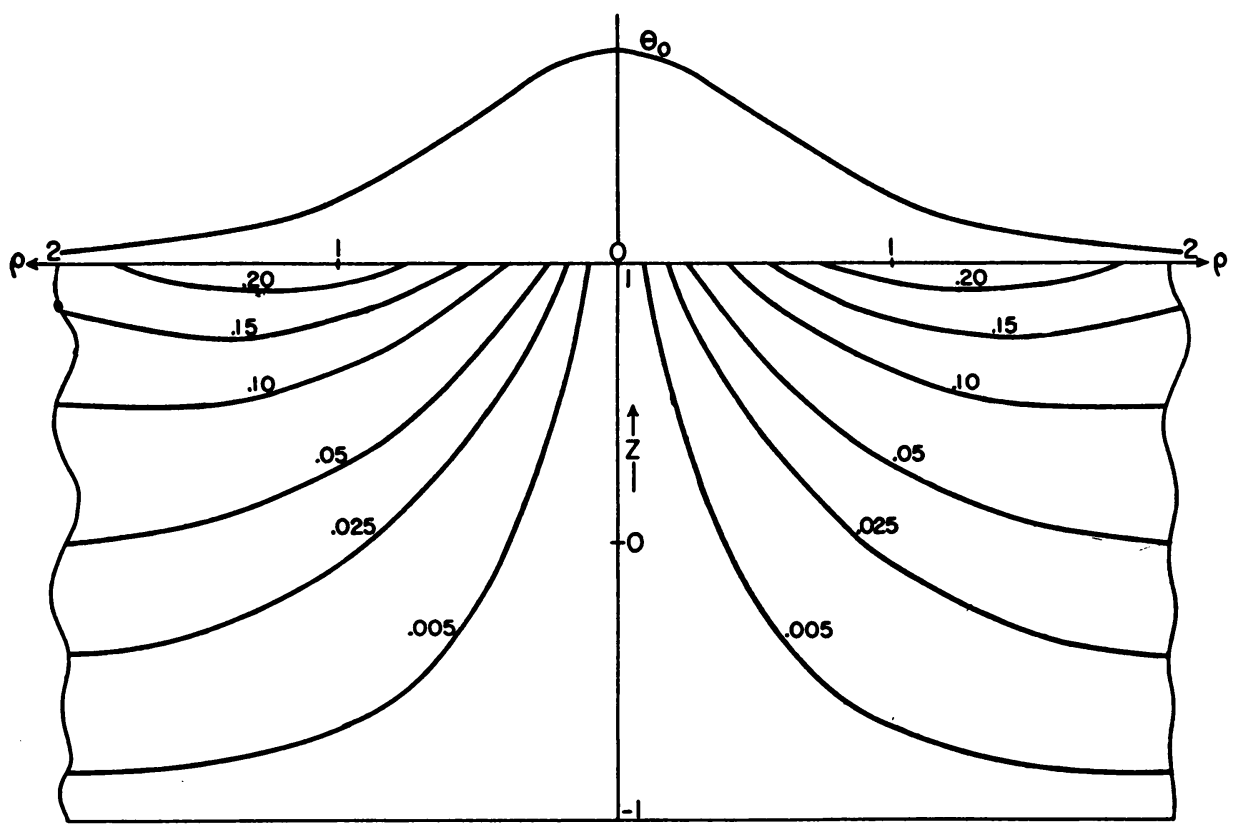

Fra. 3. Section by a plane $\vartheta=$ constant of the surfaces $\left|\sigma_{\rho}-\sigma_{\vartheta}\right|=$ constant in a thick plate. The numbers refer to the values of $8 k\left(\beta^{2}-1\right)\left(\sigma_{\vartheta}-\sigma_{\rho}\right) / b \theta_{0}\left(k^{2}-4\right)^{2}$. 
Similarly, if we make the same substitution in Eq. (26a), we find that the radial component of the displacement vector is determined by the equation

$$
\begin{aligned}
u_{\rho}=(\theta / \rho)\left\{( k + 1 + z ) \left[\rho^{2}+(k+1\right.\right. & \left.+z)^{2}\right]^{-1 / 2} \\
& \left.-(k-1-z)\left[\rho^{2}+(k-1-z)^{2}\right]^{-1 / 2}\right\},
\end{aligned}
$$

with

$$
\theta=\left(k^{2}-4\right)^{2} b \theta_{0} /\left[16 k\left(\beta^{2}-1\right)\right] .
$$

From Eq. (31) we in turn deduce that the difference of the principal stresses is

$$
\begin{aligned}
\sigma_{\diamond}-\sigma_{\vartheta}=2 \theta\left\{( k - 1 - z ) \left[\rho^{2}+\right.\right. & \left.(k-1-z)^{2}\right]^{-3 / 2} \\
& -(k+1+z)\left[\rho^{2}+(k+1+z)^{2}\right]^{-3 / 2} \\
& +2(k-1-z) \rho^{-2}\left[\rho^{2}+(k-1-z)^{2}\right]^{-1 / 2} \\
& \left.-2(k+1+z) \rho^{-2}\left[\rho^{2}+(k+1+z)^{2}\right]^{-1 / 2}\right\} .
\end{aligned}
$$

In a similar way, we find from Eq. (27a) that

$$
w=\theta\left\{\left[\rho^{2}+(k-1-z)^{2}\right]^{-1 / 2}+\left[\rho^{2}+(k+1+z)^{2}\right]^{-1 / 2}\right\} .
$$

Figure 3 shows the surface distribution of temperature in the case $k=3$, together with the sections (by a plane $\vartheta=$ constant) of the isochromatic surfaces $\left|\sigma_{\rho}-\sigma_{\vartheta}\right|=$ constant. If we had chosen a higher value of the parameter $k$ we should have obtained a surface temperature distribution which was less highly concentrated in the neighbourhood of the point $\rho=0$; on the other hand, if we had chosen a smaller value of $k$, such as $k=2.1$, the curve would have been concentrated into a small band surrounding the origin.

\section{REFERENCES}

1. E. Sternberg and E. L. McDowell, On the steady-state thermoelastic problem for the half-space, Quart. Appl. Math. 14, 381 (1957)

2. E. L. McDowell, Thermal stresses in an infinite plate of arbitrary thickness, Proc. 3rd Midwest. Conf. on Solid Mechanics, 72-85 (1957)

3. R. Muki, Thermal stresses in a semi-infinite solid and a thick plate under steady distribution of temperature, Proc. Fac. Eng. Keio. Univ. 9, 42 (1957)

4. I. N. Sneddon and D. S. Berry, The classical theory of elasticity, Handbuch der Physik 6, 1958, pp. 1-126, Sec. F

5. G. Eason, B. Noble and I. N. Sneddon, On certain integrals of Lipschitz-Hankel type involving products of Bessel functions, Phil. Trans. A 247, 529 (1955) 